\title{
Clinical Usefulness of Real-Time Polymerase Chain Reaction for the Diagnosis of Vibrio vulnificus Infection Using Skin and Soft Tissues
}

\author{
Jun-Young Lee, ${ }^{1} \dagger$ Seok Won Kim, ${ }^{2} \dagger$ Dong-Min Kim, ${ }^{3 *}$ Na Ra Yun, ${ }^{3}$ Choon-Mee Kim, ${ }^{4}$ and Sang-Hong Lee ${ }^{1}$ \\ ${ }^{1}$ Department of Orthopedic Surgery, College of Medicine, Chosun University, Gwangju, South Korea; ${ }^{2}$ Department of Neurosurgery, \\ College of Medicine, Chosun University, Gwangju, Republic of Korea; ${ }^{3}$ Division of Infectious Disease, Department of Internal Medicine, \\ College of Medicine, Chosun University, Gwangju, South Korea; ${ }^{4}$ Premedical Science, College of Medicine, Chosun University, \\ Gwangju, Republic of Korea
}

\begin{abstract}
Vibrio vulnificus is a halophilic gram-negative bacillus isolated in seawater, fish, and shellfish. Infection by $V$. vulnificus is the most severe food-borne infection reported in the United States of America. Here, we aimed to examine the clinical usefulness of polymerase chain reaction (PCR) using tissue specimens other than blood samples as a diagnostic tool for $V$. vulnificus infection. A retrospective study was conducted with patients who underwent real-time PCR of tox $R$ in both blood and skin tissues, including serum, bullae, swab, and operation room specimens, between 2006 and 2009. The median V. vulnificus DNA load of 14 patients in real-time PCR analysis of serum at the time of admission was 638.5 copies/mL blood, which was within the interquartile range (IQR: 37-3,225). In contrast, the median value by realtime PCR using the first tissue specimen at the time of admission was 16,650 copies/mL tissue fluid (IQR: 4,419-832,500). This difference was statistically significant $(P=0.022)$. DNA copy numbers in tissues were less affected by short-term antibiotic administration than that in blood samples, and antibiotic administration increased the DNA copy number in some patients. We found, for the first time, that DNA copy numbers in tissues of patients infected by V. vulnificus were higher than those in blood samples. Additionally, skin lesions were more useful than blood samples as specimens for PCR analysis in patients administered antibiotics for $V$. vulnificus infection before admission.
\end{abstract}

\section{INTRODUCTION}

Vibrio vulnificus is a halophilic gram-negative bacillus that can be isolated from seawater, fish, and shellfish. Consumption of raw fish and shells that are contaminated or exposure to contaminated seawater results in $V$. vulnificus infection, which can cause primary septicemia or wound infection and gastroenteritis. ${ }^{1}$ Vibrio vulnificus infection is the most severe disease among food-borne infections reported in the United States of America. ${ }^{2}$

Rapid and appropriate initial antimicrobial treatment can reduce mortality caused by $V$. vulnificus infection; therefore, rapid diagnosis is critical for achieving good clinical outcomes. ${ }^{3}$ Because V. vulnificus grows well in standard blood culture medium or nonselective medium (such as blood agar) routinely used for wound cultures, $V$. vulnificus is easily diagnosed after it has been cultured. However, $V$. vulnificus is also susceptible to most antibiotics and can be highly affected by antibiotic administration, ${ }^{4}$ which may complicate diagnosis. Polymerase chain reaction (PCR) has been used for the rapid diagnosis of $V$. vulnificus by targeting $V$. vulnificus-specific genes. In this respect, realtime PCR has been reported as a highly sensitive and specific diagnostic method for $V$. vulnificus infection in clinical practice. . $^{5}$

Although quantification using real-time PCR has been shown to be correlated with disease severity or mortality in patients with $V$. vulnificus infection, ${ }^{7}$ no studies have examined the clinical utility of PCR using tissue specimens other than blood samples. Accordingly, in this study, we aimed to investigate the clinical usefulness of real-time PCR using

${ }^{*}$ Address correspondence to Dong-Min Kim, Department of Internal Medicine, College of Medicine, Chosun University, 588 Seosuk-dong, Dong-gu, Gwangju 501-717, Republic of Korea. E-mail: drongkim@ chosun.ac.kr

† These authors contributed equally to this work. bullae, swab, and surgical specimens for patients suspected to have $V$. vulnificus infection.

\section{MATERIALS AND METHODS}

Of patients admitted to Chosun University Hospital between 2006 and 2009 who had real-time PCR testing for targeting of the $V$. vulnificus-specific tox $R$ as previously described, ${ }^{6}$ including prospective research data on adults 18 years or older who had skin and soft tissue infections, such as cellultitis or necrotizing fasciitis, and who had blood and skin tissue culture tests, ${ }^{8}$ patients with skin and soft tissue infection who had real-time PCR analysis of both serum and skin tissues, including bullae, swab, and surgical specimens, at the time of admission were subjected to retrospective chart analysis of the presence or absence or antibiotic administration before admission. ${ }^{5,6}$ Bacterial species were identified using a VITEK 2 automated system (BioMérieux, Marcy-L'Etoile, France) in our hospital.

The study was approved by the Ethics in Human Research Committee of Chosun and Chonnam University Hospital.

Statistical analyses of all test items were carried out using Statistical Package for the Social Sciences (SPSS) for Windows, version 17.0 (SPSS Korea, Seoul, Korea). Vibrio vulnificus DNA copy numbers in serum and tissues, such as bullae and surgical specimens, were compared using the Wilcoxon signed rank test, a nonparametric paired $t$ test. Differences with $P$ values of below 0.05 were considered statistically significant.

\section{RESULTS}

A total of 14 patients were found to have at least one positive result from real-time PCR with both skin and blood specimens. Of these patients, the mean age was 58.9 years, and all but one patient was male (Table 1 ). 
LEE AND OTHERS

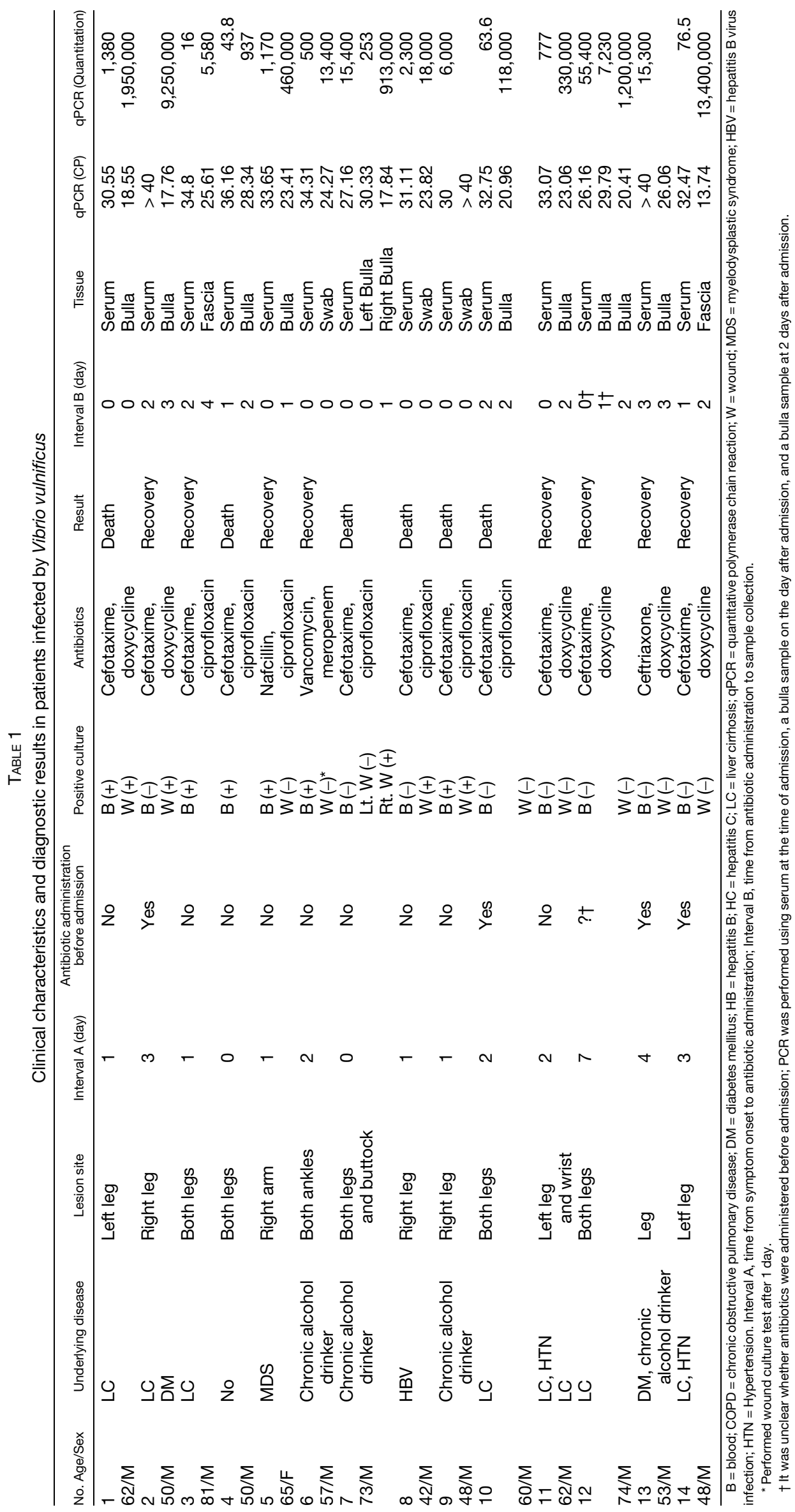


Six of the 14 patients died (mortality rate: $42.9 \%$ ). Of the 14 patients, bacteria were identified in blood or skin specimens from nine patients, and bacteria were cultured in both skin and blood samples from two of these nine patients and in either skin or blood samples from seven of these nine patients (four patients had blood cultures only and three patients had skin cultures only). Of the six patients whose blood culture tests identified $V$. vulnificus at the time of admission, three patients had follow-up blood culture tests around 24 hours, which did not yield cultured bacteria. In real-time PCR analysis of serum samples collected at the time of admission, the median $V$. vulnificus DNA load of 14 patients was 638.5 copies/mL blood (interquartile range [IQR]: $37-3,225)$. In contrast, the median real-time PCR value using the first tissue specimen at the time of admission was 16,650 copies $/ \mathrm{mL}$ tissue fluid (IQR: 4,419-832,500); this different was statistically significant $(P=0.022)$.

Of the 14 patients in this study, four had received antibiotics before admission, and nine were admitted without antibiotic administration; the status of the final patient was not clear. The mean serum DNA copy number of patients without antibiotic administration was 9,134 $\pm 18,028$ copies/mL, whereas that of patients with antibiotic administration before admission was $35 \pm 41$ copies $/ \mathrm{mL}$. Additionally, the DNA copy number in tissues of patients without antibiotic administration was 272,822 \pm 646,606 copies $/ \mathrm{mL}$, whereas that of patients with antibiotic administration was $5,695,825 \pm 3,358,658$ copies $/ \mathrm{mL}$.

Two patients (cases 2 and 13) were positive by real-time PCR using bullae samples but negative using blood samples. These patients had undergone antibiotic administration for 2 or 3 days before admission, respectively, and were then transferred to our hospital. In patient 2, blood culture tests did not identify any bacteria; however, culture tests with aspiration of bulla yielded cultured bacteria, and the DNA copy number was 9,250,000 copies/mL tissue fluid, showing a high copy number even after administration of antibiotics for 2 days. Patient 13 was treated for cellulitis at a local clinic for 3 days and was then transferred for bullae developed; in this patient, both skin and blood cultures were negative, and $V$. vulnificus DNA was not detected by PCR using blood samples but was detected (at 15,300 copies/mL) in bulla aspirates, despite the lack of detection of cultured bacteria from bulla aspirates.

Of the six patients whose blood samples were shown to have $V$. vulnificus in culture, no patients had received antibiotics for 24 hours or more prior to undergoing blood culture tests.

Of the five patients $(10-14)$ for whom $V$. vulnificus was not identified in blood or skin samples, three patients had undergone antibiotic administration before admission, and one patient, for whom it was not clear whether antibiotics had been administered, was admitted after treatment at a local clinic 1 day before admission. Although $V$. vulnificus was not identified in blood and skin samples from these five patients, high DNA copy numbers were identified in the blood or bullae, particularly in bullae (Table 1).

For case 12, the DNA copy number from real-time PCR using bullae 1 day after admission was 7,230 copies/mL; this number increased to $1,200,000$ copies $/ \mathrm{mL}$ in bullae tests performed after an additional day of antibiotic administration. For case 7, 253 DNA copies/mL were identified in bullae of the left leg in PCR analysis performed immediately after admission, whereas the copy number in the bullae of the right leg increased to 913,000 copies/mL after antibiotics were administered for an additional day.

\section{DISCUSSION}

Vibrio sepsis induced by $V$. vulnificus is a severe disease that causes rapidly progressing septic shock syndrome and is associated with mortality rates of $50 \%$ or more within a few days. Therefore, in cases of $V$. vulnificus infection, it is critical to administer antibiotics with a high antibacterial activity against $V$. vulnificus soon after rapid diagnosis. ${ }^{8,9}$ Because real-time PCR results can be obtained within 2 hours of testing, PCR can be used for rapid diagnosis. tox $R$, which is often used as a target gene, encodes a transmembrane DNA binding regulatory protein in Vibrio species and has been shown to have $100 \%$ specificity for Vibrio species in real-time PCR analyses. ${ }^{5,6}$ Moreover, in previous studies, PCR of toxR was shown to yield higher DNA copy numbers in patients who died of $V$. vulnificus sepsis than in patients who survived; thus, high DNA copy number by PCR is a clinically useful predictive factor for prognosis during the early stage of $V$. vulnificus infection. ${ }^{7}$ However, although skin or blood cultures should be performed during the early stages of infection for rapid diagnosis, many patients with Vibrio sepsis are admitted after antibiotic administration, and no studies have examined the usefulness of skin lesions as specimens for culture or PCR tests. Accordingly, in this study, we used blood and skin samples to determine the utility of skin samples as PCR specimens in the early diagnosis of $V$. vulnificus infection.

Of the six patients who were found to have $V$. vulnificus in blood cultures at the time of admission, follow-up blood culture tests were performed for three patients after 24 hours, yielding no bacteria. Moreover, according to a chart data analysis of 23 patients in whom $V$. vulnificus was identified in blood culture tests after admission to our hospital, eight patients had additional blood culture tests performed about 24 hours after antibiotic administration; these data showed that no patients remained positive for $V$. vulnificus after 24 hours (data not shown). Therefore, it is highly unlikely that the bacteria could be identified from blood culture tests in patients who have already received antibiotics, particularly tetracyclines or quinolones, before admission.

In the present study, a total of six patients were either admitted after antibiotic administration or had PCR tests performed using serum specimens after antibiotic administration. Of these patients, four were found to have $V$. vulnificus DNA in serum samples. Moreover, nine patients who underwent PCR using tissues and had received antibiotics before and after admission showed considerable numbers of DNA copies, even after antibiotic administration. Interestingly, cases 7 and 12 had higher DNA copy numbers in PCR tests performed after 1 and 2 days of antibiotic administration at our hospital than at initial admission. Therefore, these data showed that specimens from patients who were admitted after administration of antibiotics, particularly bullae or surgical specimens, were useful for diagnosis by PCR. 
The present study showed that tissues had higher DNA copy numbers than blood samples. Therefore, we suggest that $V$. vulnificus may accumulate in lesions within tissues rather than in the blood. From our data, we indirectly speculated that tissues may have higher amounts of bacteria than blood. Additional studies comparing the amounts of bacteria present in blood and tissue samples are needed. Moreover, studies should clarify whether more DNA may be released immediately after antibiotic administration in tissue.

In conclusion, the present study showed, for the first time, that there were higher $V$. vulnificus DNA copy numbers in tissue samples than in blood samples from patients infected with $V$. vulnificus. Additionally, our findings suggested that skin lesions were more useful than blood samples for PCR-based diagnosis of $V$. vulnificus infection in patients admitted after administration of antibiotics active against $V$. vulnificus.

Received June 22, 2016. Accepted for publication February 13, 2017.

Published online May 8, 2017.

Financial support: This work was supported by a grant from the Clinical Medicine Research Institute of the Chosun University Hospital (2015).

Authors' addresses: Jun-Young Lee, Sang-Hong Lee, Department of Orthopaedic Surgery, College of Medicine, Chosun University, Dong-Gu, Gwangju, Republic of Korea, E-mail: leejy88@chosun. ac.kr, shalee@chosun.ac.kr. Seok-Won Kim, Department of Neurosurgery, College of Medicine, Chosun University, Gwangju, Korea, E-mail: chosunns@chosun.ac.kr. Dong-Min Kim, and Na-Ra Yun, Department of Internal Medicine, College of Medicine, Chosun University, Dong-Gu, Gwangju, Republic of Korea, E-mail: drongkim@ chosun.ac.kr, shine@chosun.ac.kr. Choon-Mee Kim, Premedical
Science, College of Medicine, Chosun University, Gwangju, South Korea, E-mail: choonmee@chosun.ac.kr.

\section{REFERENCES}

1. Haq SM, Dayal HH, 2005. Chronic liver disease and consumption of raw oysters: a potentially lethal combination: a review of Vibrio vulnificus septicemia. Am J Gastroenterol 100: 1195-1199.

2. Mead PS, Slutsker L, Dietz V, McCaig LF, Bresee JS, Shapiro C, Griffin PM, Tauxe RV, 1999. Food-related illness and death in the United States. Emerg Infect Dis 5: 607-625.

3. Klontz KC, Lieb S, Schreiber M, Janowski HT, Baldy LM, Gunn RA, 1988. Syndromes of Vibrio vulnificus infections. Clinical and epidemiologic features in Florida cases, 1981-1987. Ann Intern Med 109: 318-323.

4. Kim DM, Lym Y, Jang SJ, Han H, Kim YG, Chung CH, Hong SP, 2005. In vitro efficacy of the combination of ciprofloxacin and cefotaxime against Vibrio vulnificus. Antimicrob Agents Chemother 49: 3489-3491.

5. Takahashi H, Hara-Kudo Y, Miyasaka J, Kumagai S, Konuma H, 2005. Development of a quantitative real-time polymerase chain reaction targeted to the toxR for detection of Vibrio vulnificus. J Microbiol Methods 61: 77-85.

6. Kim HS, Kim DM, Neupane GP, Lee YM, Yang NW, Jang SJ, Jung SI, Park KH, Park HR, Lee CS, Lee SH, 2008. Comparison of conventional, nested, and real-time PCR assays for rapid and accurate detection of Vibrio vulnificus. J Clin Microbiol 46: 2992-2998.

7. Kim DM, Jung SI, Jang HC, Lee CS, Lee SH, Yun NR, Neupane GP, Park KH, 2011. Vibrio vulnificus DNA load and mortality. J Clin Microbiol 49: 413-415.

8. Ho YC, Chang SC, Lin SR, Wang WK, 2009. High levels of mecA DNA detected by a quantitative real-time PCR assay are associated with mortality in patients with methicillin-resistant Staphylococcus aureus bacteremia. J Clin Microbiol 47: 1443-1451.

9. Yun NR, Kim DM, Lee J, Han MA, 2015. pH level as a marker for predicting death among patients with Vibrio vulnificus infection, South Korea, 2000-2011. Emerg Infect Dis 21: 259-264. 